\title{
The value of delaying hCG administration to enable maturation of medium-sized follicles in patients undergoing superovulation for IVF/ICSI
}

\author{
Awoniyi O. Awonuga ${ }^{1} \cdot$ Karen Wheeler $^{1} \cdot$ Mili Thakur $^{1,2} \cdot$ Roohi Jeelani $^{1}$. \\ Michael P. Diamond $^{3}$ - Elizabeth E. Puscheck ${ }^{1}$
}

Received: 1 April 2017 / Accepted: 20 September 2017 /Published online: 30 September 2017

(C) Springer Science+Business Media, LLC 2017

\begin{abstract}
Purpose The purpose of the study is to determine whether continued stimulation of mature follicles to allow "catch up" growth of medium-sized follicles in assisted reproductive technology compromises the clinical pregnancy (CPR) and live birth (LBR) rates in IVF/ICSI cycles.

Methods This retrospective cohort study reviewed 200 first IVF \pm ICSI cycles out of a total of 340 cycles with complete data. Women underwent stimulation protocols with gonadotropins $(\mathrm{Gn})$ and GnRH antagonist. Treatment cycles were divided into two groups (Gp): hCG administration delayed despite the presence of two mature follicles, defined as $\geq 18 \mathrm{~mm}[\mathrm{Gp} 1, n=79]$ and hCG administration given when there were two mature follicles [Gp2, $n=121]$.

Results The patients in Gp1 were significantly younger than those in $\mathrm{Gp} 2[32.9$ (4.5) vs. 34.3 (4.8), $p=0.04]$ and needed a median of one more day of superovulation before ovulation was triggered with hCG. The extra days was associated with the use of 450 [75-2025] more Gn, such that at the time the hCG was administered, patient's in group 1 had developed significantly greater number of follicles $\geq 18 \mathrm{~mm}$ [mean (SD), 4.9 (1.8) vs. 3.4 (1.7), $p<0.0001]$. The clinical
\end{abstract}

Awoniyi O. Awonuga

aawonuga@med.wayne.edu; niyiawonuga@aol.com

1 Division of Reproductive Endocrinology and Infertility, Department of Obstetrics and Gynecology, Wayne State University School of Medicine/Detroit Medical Center, 275 E. Hancock Street, Detroit, MI 48201, USA

2 Division of Genetic and Metabolic Disorders, Department of Pediatrics and Center for Molecular Medicine and Genetics, Wayne State University School of Medicine, Detroit, MI, USA

3 Department of Obstetrics and Gynecology, Augusta University, Augusta, GA, USA pregnancy (48.1 vs. $38.0 \%$, [OR (95\% CI)] [1.6 (1.0-2.5), $p=0.09])$ and live birth (43.0 vs. 35.5\%, [1.4 (0.9-2.3), $p=0.21]$ ) rates per cycle started were not significantly different between the two groups. Forward stepwise logistic regression showed that only maternal age $(p=0.04)$ influenced clinical pregnancy rates $(\mathrm{OR}=0.88$, CI $0.78-0.99)$ and only the number of days for superovulation influenced live birth rates $(\mathrm{OR}=0.65$, CI $0.486-0.869)$.

Conclusion This study demonstrated that delaying hCG administration to allow further growth of the medium-sized follicles added further days of superovulation and cost without improvement in CPR and LBR.

Keywords IVF stimulation $\cdot$ Superovulation $\cdot$ hCG . Follicles · Oocyte retrieval

\section{Introduction}

The tremendous improvement in the success rates of assisted reproductive technology (ART) cycles, despite the transfer of increasingly fewer embryos [1], can be attributed to better superovulation protocols, embryo culture media, and embryo transfer technique. Nevertheless, the pregnancy rates still vary between infertility centers, thus warranting measures to maximize success rates. The number of oocytes collected during ART is one of the most important determinants of overall success. However, there is considerable variability in the criteria used for inducing final oocyte maturation in IVF, which reflects on the fact that the basis for such practice in part is both arbitrary and empirical.

Following superovulation, ovulation triggering is often advised when more than two or three follicles $\geq 18 \mathrm{~mm}$ have developed on ultrasound $[2,3]$. Continued stimulation to allow medium-sized follicles to grow further may increase the 
number of oocytes retrieved; however, such practice may adversely affect endometrial receptivity [3] or predispose patients to developing ovarian hyperstimulation syndrome (OHSS). The point at which the advantages of multifollicular development outweigh potential complications such as OHSS is difficult to determine [4]. Although OHSS is more likely to occur with higher estradiol levels on the day of hCG administration, this is not invariable [5]. Two studies have suggested that superovulation, with the intent to collect more oocytes to improve pregnancy and live birth rates, is futile when greater than 15 oocytes are retrieved $[4,6]$.

Tan and colleagues [7] evaluated the impact of delaying ovulation triggering for 1 or 2 days when criteria for hCG were met (mean diameter of the largest follicle had reached $18 \mathrm{~mm}$, and at least two other follicles were greater than $14 \mathrm{~mm}$ ) in a randomized study and found no significant differences in the clinical pregnancy rates. However, the follicular criteria used to decide when hCG was given in the study by Tan and colleagues [7] were conservative (mean diameter of the largest follicle of $18 \mathrm{~mm}$ and at least two other follicles $>14 \mathrm{~mm}$ ), and hCG injections were delayed for a maximum of 2 days. Nevertheless, the ability to vary the timing of hCG administration may also allow greater flexibility in scheduling of oocyte collection to meet logistical needs of the patient and her partner as well as the ART center, without compromising the clinical pregnancy rates (CPRs) [8-12]. Reproductive endocrinologists sometimes sacrifice lead follicle(s) when the remaining cohort is further behind to allow additional follicles to reach maturity. It remains to be determined, however, whether continued stimulation of mature follicles to allow "catch up" growth of medium-sized follicles (14-16 mm) in ART compromises the CPR and live birth rates (LBRs) in IVF/ICSI cycles. The objective of this study was to determine whether continued stimulation of mature follicles to allow "catch up" growth of medium-sized follicles in ART compromises the CPR and LBR in IVF/ICSI cycles.

\section{Materials and methods}

Following approval from the Wayne State University Institutional Review Board, we undertook a retrospective analysis of 340 consecutive IVF \pm ICSI cycles in 240 patients who achieved the development of at least two or more follicles (>18 mm) between January 2003 and December 2009. Of these, 237 patients who underwent their first IVF/ICSI treatment at our institution were reviewed of which 37 cycles in 37 patients were excluded. The reasons for their exclusion are enumerated in Fig. 1. Women underwent standard stimulation protocols with gonadotropins $(\mathrm{Gn})$ (Gonal-F®, Serono; and Menopur ${ }^{\circledR}$ or Repronex ${ }^{\circledR}$, Ferring) and GnRH antagonist for pituitary down-regulation (Ganirelix acetate, Organon). Downregulation with $\mathrm{GnRH}$ antagonists is the standard protocol in our institution. Patients were started on oral contraceptives for 14-30 days prior to and were stopped 3 days before starting ovarian stimulation. GnRH antagonist ( $0.25 \mathrm{mg}$ daily) was added when at least one follicle reached $14 \mathrm{~mm}$ in diameter and was continued until day of hCG administration. An additional $75 \mathrm{IU}$ of $\mathrm{LH}$ and FSH (Menopur® or Repronex ${ }^{\circledR}$ ) was added to the Gn. regimen when GnRH antagonist was started. At our center, the criteria to proceed to oocyte aspiration are the presence of at least four mature follicles between 16 and $20 \mathrm{~mm}$ in size with a minimum of two follicles $18 \mathrm{~mm}$ or greater. Treatment cycles were divided into two groups (Gp), based on the day the patients first met the criteria for hCG administration. hCG administration was delayed despite patients having met these criteria [Gp1,n=79] and hCG administration given when these criteria were met [Gp2, $n=121]$. The decision to administer or withhold hCG and for how long was based solely on the attending physician's discretion after reviewing the stimulation parameters. For the purpose of this study, the number of days that elapsed before hCG was eventually given was extracted and recorded. Oocyte collection was performed $36 \mathrm{~h}$ after hCG trigger. Embryo transfer was performed 2 to 5 days after oocyte collection. Implantation rate was defined as the number of gestation sacs per embryo transferred, while CPR was defined as the number of cycles with intrauterine gestational $\operatorname{sac}(\mathrm{s})$ with fetal heart pulsation at 4-5 weeks from the day of transfer. Live birth rate was defined as the number of pregnancies that resulted in live births per cycle started.

All statistical analyses were performed using Statistical Package for Social Sciences (SPSS, Version 18 for Windows; SPSS, Inc., Chicago, IL, USA). All tests were conducted using $p$ value less than 0.05 for statistical significance. The data were expressed as mean + standard deviation (SD), and median [range] for continuous variables, and the number of cases $(n)$ and percentage of occurrence (\%) for categorical variables. Between group, differences were analyzed with chisquare test and Fisher's exact test for categorical data, and Student's $t$ test and Mann-Whitney $U$ test for continuous variables as appropriate. We used binary logistic regressions to model CPRs and LBRs with forward stepwise selection according to the likelihood ratio method based on inclusion criteria of $p \leq 0.05-p \leq 0.10$. The variables of maternal age, serum estradiol when decision was made to delay hCG administration, the total number of days of superovulation, the number of follicles $\geq 18 \mathrm{~mm}$ on day of $\mathrm{hCG}$, the size of the lead follicle and the day of embryo transfer (Tables 1, 2, and 3), and whether the cycle was delayed or not delayed were included in the model. Statistical significance was defined as $p<0.05$. 
Fig. 1 Depicts the number of cycles included and excluded from analysis

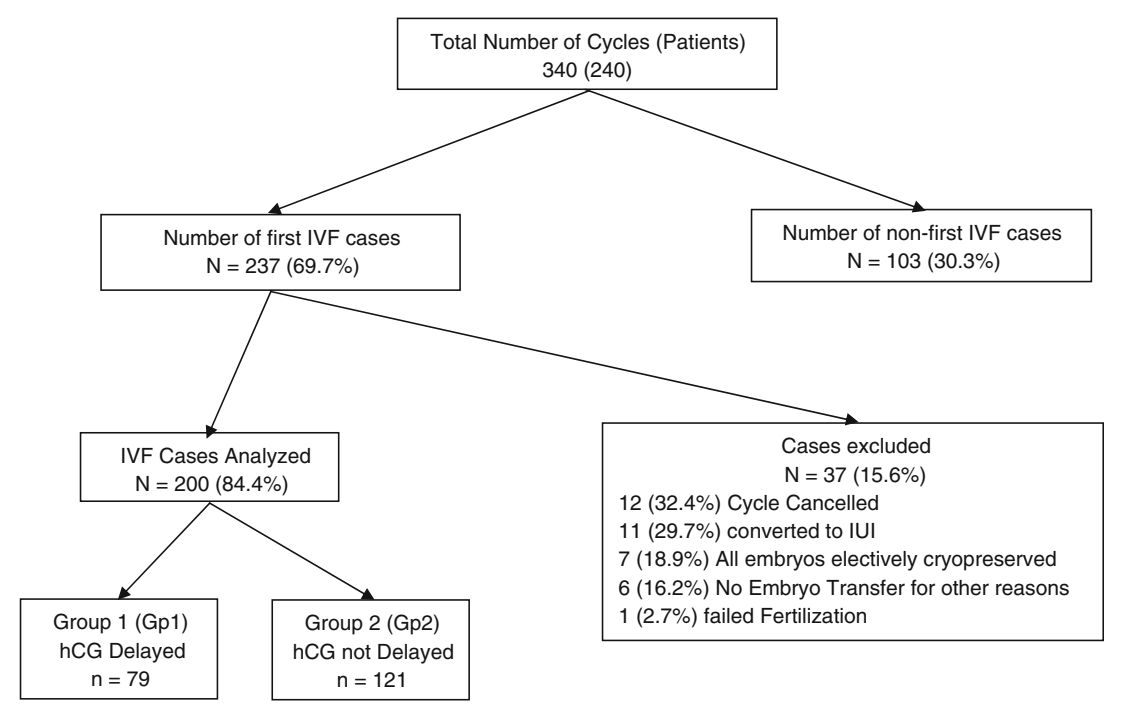

\section{Results}

During the study period, 237 women underwent their first IVF \pm ICSI cycle. Of these, 37 cycles were excluded from further analyses and the reasons for exclusion are enumerated in Fig. 1. Of the remaining 200 cycles, 79 had hCG administration withheld despite the presence of 4 follicles $\geq 16 \mathrm{~mm}$ when 2 were $\geq 18 \mathrm{~mm}$ and in 121 cycles, hCG was given when these criteria were met. The overall clinical pregnancy (intrauterine sac and fetal heart activity) and livebirth rates per cycle started were 48.1 and $38.0 \%$, respectively. Among patients who had their hCG administration delayed, a greater proportion, 68 (86.1\%) needed 2 further days of superovulation, 4 (5.1\%) needed 1 more day, 6 (7.6\%), and $1(1.3 \%)$ needed 3 and 4 more days, respectively. However, the clinical pregnancy $(p=0.37)$ and the livebirth $(p=0.28)$ rates did not differ in relation to the number of days the patient was delayed before hCG trigger was administered. The CPR (48.1 vs. $38.0 \%)$ and
Table 1 Baseline characteristics of patients in whom hCG administration was delayed (group 1) compared with those not delayed (group 2)

\begin{tabular}{|c|c|c|c|}
\hline & $\begin{array}{l}\text { Group } 1 \\
n=79\end{array}$ & $\begin{array}{l}\text { Group } 2 \\
n=121\end{array}$ & $p$ \\
\hline Age (years) & $\begin{array}{l}32.9(4.5) \\
33.0[20-43]\end{array}$ & $\begin{array}{l}34.3(4.8) \\
34[17-44]\end{array}$ & 0.04 \\
\hline BMI $\left(\mathrm{kg} / \mathrm{m}^{2}\right)$ & $27.0(5.9)$ & $27.9(6.0)$ & 0.27 \\
\hline Duration of infertility (months) & 30.0 [4-132] & $36[5-204]$ & 0.19 \\
\hline Gravidity $[n(\%)]$ & & & 0.46 \\
\hline $\begin{array}{l}0 \\
1-2\end{array}$ & $\begin{array}{l}44(55.7) \\
29(36.7)\end{array}$ & $\begin{array}{l}59(48.8) \\
47(38.8)\end{array}$ & \\
\hline$\geq 3$ & $6(7.6)$ & $15(12.4)$ & \\
\hline Parity $[n(\%)]$ & & & 0.25 \\
\hline 0 & $62(78.5)$ & 87 (71.9) & \\
\hline $1-2$ & $14(17.7)$ & $32(26.4)$ & \\
\hline$\geq 3$ & $3(3.8)$ & $2(1.7)$ & \\
\hline Etiology of infertility $[n(\%)]$ & & & 0.90 \\
\hline Male factor & $43(54.4)$ & $60(49.6)$ & \\
\hline Tubal & $15(19.0)$ & $28(23.1)$ & \\
\hline Ovulatory dysfunction & $14(17.7)$ & $19(15.7)$ & \\
\hline Unexplained & $4(5.1)$ & $8(6.6)$ & \\
\hline Others & $3(4.5)$ & $6(5.0)$ & \\
\hline Day 3 FSH (mIU/L) & $7.5(2.4)$ & $7.1(3.3)$ & 0.53 \\
\hline Day 3 estradiol (pg/mL) & $41.1[11.0-169.0]$ & $41.3[20.0-249.0]$ & 0.83 \\
\hline Antral follicle count & $13[2-40]$ & $11[0-43]$ & 0.15 \\
\hline
\end{tabular}

Unless otherwise stated, results are mean (SD) or median [range] 
Table 2 Superovulation parameters in patients in whom hCG administration was delayed (group 1) compared with those not delayed (group 2)

\begin{tabular}{llll}
\hline & $\begin{array}{l}\text { Group 1 } \\
n=79\end{array}$ & $\begin{array}{l}\text { Group 2 } \\
n=121\end{array}$ & $p$ \\
\hline Parameters when hCG withheld & hCG withheld & hCG not withheld & \\
E2 at decision to delay (pg/mL) & $1692.3(728.1)$ & $2431.3(1012.3)$ & $<0.0001$ \\
Follicles 14-17 mm & $6.1(2.8), 6[0-14]$ & $12.0(7.5), 6[0-34]$ & $<0.0001$ \\
Follicles 17 mm & $1.5(1.0), 1[0-5]$ & $1.6(1.3), 2[0-8]$ & 0.43 \\
Follicles > 18 & $2.0(1.0), 2[0-4]$ & $3.5(1.6), 3[0-13]$ & $<0.0001$ \\
Extra days on Antagonist & $2.1(0.4), 2[1-4]$ & & \\
Extra days on Gn & $1.4(0.5), 2[1-4]$ & & 0.30 \\
Extra dose of Gn used (IU) & $450[75-2025]$ & & 0.09 \\
Parameters on the day hCG was administered & & & 0.93 \\
Gn (IU) used for superovulation & $3563[1238-14,625]$ & $3525[1162-17,025]$ & \\
Number of days of superovulation & $9.9(1.8)$ & $10.3(1.8)$ & 0.29 \\
Serum estradiol (pg/mL) & $2443.9(830.1)$ & $2436.8(1013.0)$ & $<0.0001$ \\
Total number of follicles & $20.2(10.0)$ & $21.9(10.8)$ & $<0.0001$ \\
Number of follicles $>18$ mm & $5.0(1.9)$ & $3.4(1.6)$ & $<0.0001$ \\
Number of follicles 14-17 mm & $6.1(2.9)$ & $13.0(6.6)$ & 0.12 \\
Size lead follicle (mm) & $22.3(2.3)$ & $20.2(1.6)$ & 0.78 \\
Endometrium thickness (mm) & $10.6(2.4)$ & $10.0(2.1)$ & $10.7(1.9)$ \\
Day hCG trigger was given & $10.7(1.7)$ & & \\
\hline
\end{tabular}

Unless otherwise stated, data are in mean (standard deviation) and median [range]

$n=$ number

LBR (43.0 vs, $35.5 \%)$ were higher in the delayed compared with the non-delayed group; however, the odds ratio [OR $(95 \% \mathrm{CI})]$ of achieving a clinical pregnancy [1.56 (1.00$2.52)]$ and live birth [1.39 (0.85-2.27)] was not significantly different. Apart from the age of patients, there were no significant differences in demographic variables (body mass index, duration or etiology of infertility, gravidity, and parity) and baseline measurements (day 3 serum FSH, $\mathrm{E}_{2}$, and antral follicle count) between the two groups (Table 1).

At the time hCG was administered to patients in group 2 and withheld in patients in group 1, the mean serum estradiol, and number of follicles that measured $14-17 \mathrm{~mm}$ and those that were $>18 \mathrm{~mm}$ were significantly lower in group $1 \mathrm{com}-$ pared with group $2(p<0.0001)$ (Table 2$)$. The patients in group 1 needed a median of two [1-4] extra days of antagonist, and one [1-4] more day of superovulation, which was associated with the use of 450 IU [75-2025] more of Gn, to catch up and overtake their group 2 counterparts in the number of follicles $\geq 18 \mathrm{~mm}$ at the time of hCG. However, on the day hCG trigger was administered, the total dose of Gn used for superovulation in group 1 compared to that in group 2 was not statistically significant. Also, not significantly different on the
Table 3 Treatment outcome in patients in whom hCG administration was delayed (group 1) compared with those not delayed (group 2)

\begin{tabular}{llll}
\hline & $\begin{array}{l}\text { Group 1 } \\
n=79\end{array}$ & $\begin{array}{l}\text { Group 2 } \\
n=121\end{array}$ & $p$ \\
\hline Number of oocytes collected & $15[4-34]$ & $14[2-40]$ & 0.89 \\
Percentage of oocyte collected per follicle aspirated & $72.0(21.1)$ & $69.2(21.0)$ & 0.38 \\
Percentage of mature oocytes per oocyte collected & $80.5(14.2)$ & $81.1(15.4)$ & 0.76 \\
Fertilization rate & $61.5(18.6)$ & $62.2(20.0)$ & 0.79 \\
Number of embryo(s) transferred & $2.9(0.9)$ & $2.8(1.1)$ & 0.82 \\
Number of embryos cryopreserved & $5[0-32]$ & $4[0-27]$ & 0.46 \\
Day of embryo transfer & $3.1(0.6)$ & $3.0(0.4)$ & 0.10 \\
Moderate/severe OHSS $[n,(\%)]$ & $3(3.8)$ & $0(0.0)$ & 0.06 \\
CPR per cycle $[n,(\%)]$ & $38(48.1)$ & $46(38.0)$ & 0.19 \\
Live birth per cycle $[n,(\%)]$ & $34(43.0)$ & $43(35.5)$ & 0.30 \\
\hline
\end{tabular}

Unless otherwise stated, data are in mean (standard deviation) and median [range] $n=$ number 
day of hCG trigger were the numbers of days required for superovulation, the total number of follicles that developed, and the $\mathrm{E}_{2}$ levels. However, the lead follicle was significantly larger $(p<0.0001)$ and the number of follicles $\geq 18 \mathrm{~mm}$ significantly higher in group 1 compared with that in group 2 (Table 2). Consistent with the development of a comparable total number of follicles, the number of oocytes collected and the percentage that were mature, fertilization rates, and the number of embryos transferred or cryopreserved were not significantly different between the two groups. Similarly, the CPR and LBR were not significantly different between the two groups (Table 3). However, when we performed forward stepwise logistic regression in order to determine whether any of the significant differences seen between the two groups influenced the CPRs and LBRs, only maternal age $(p=0.04)$ influenced CPRs (OR $=0.88$, CI $0.78-0.99)$ and only the number of days for superovulation influenced LBRs $(\mathrm{OR}=0.65$, CI $0.486-0.869)$.

\section{Discussion}

In the present study, delaying hCG to allow further growth of medium-sized follicles did not result in any statistically significant difference in either clinical pregnancy rate or live birth rate. Logistic regression analysis controlling for confounding variables suggest that the only factor contributing to differences in CPR was maternal age, while only the length of superovulation influenced the LBR. Our data suggest that for each one-unit increase in maternal age, there is a $12 \%$ decrease in the odds of clinical pregnancy, and for each additional day of superovulation, there was a 35\% decrease in the odds of a live birth. A major challenge in reproductive endocrinology has been to develop stimulation protocols that will "ideally" synchronize the development of a mature cohort of follicles. However, the advent of GnRH agonists [13], and antagonists [14], to prevent spontaneous LH surges and spontaneous ovulation has allowed flexibility in the timing of hCG administration. These have resulted in greater flexibility in allowing further growth of medium-sized follicles before hCG administration. Furthermore, the recovery of multiple fertilizable oocytes allows for cryopreservation of extra or surplus embryos, with the consequent reduction in the risk of multiple pregnancies and the improvement in the cumulative pregnancy rate following IVF cycles. In the present study, however, the number of embryos cryopreserved did not defer between those that had compared with dose that did not have their hCG trigger delayed.

In the present study, all patients underwent stimulation protocol using $\mathrm{Gn}$ and GnRH antagonist. There was no statistical significant difference in demographics apart from maternal age, the ovarian reserve parameters, and the etiology of infertility. Similar to previous studies on delaying hCG administration, the total number of follicles that developed, the number of oocytes collected [7, 15], fertilization rates, and the number of embryos transferred were not significantly different between groups [7, 16]. Whereas, others [16] have found that a significantly higher dose of $\mathrm{Gn}$ was required for superovulation with a corresponding higher $\mathrm{E}_{2}$ levels on the day of hCG in the delayed group; we found no such association in this study. The findings in our study are at variance with those of Tan et al. [7] who found similar pregnancy rates in patients whose stimulation continued for 1 and 2 days. Similarly, unlike the assertion by Clark et al. [15] that prolongation of follicular stimulation by 1 day and Kolibianakis et al. [16], by 2 days, significantly reduced the CPR, we found that only maternal age decreased CPR. The present study represents an advancement of the work by Tan and colleagues [7] as their study contained a relatively small number of patients (79, 84 and 84 in their 3 groups); the criteria for giving hCG was conservative ( 3 follicles measuring $>14 \mathrm{~mm}$ when the largest is $18 \mathrm{~mm}$ ) and hCG injections were delayed for a maximum of 2 days. Our study also advances on the publications by Clark et al. [15] and Kolibianakis et al. [16], as we reported on live birth rate and we showed that only duration of superovulation influenced the LBRs.

The differences between our study and those of others could be attributed to the use of different stimulation protocols [15]. Even with the use of Gn and GnRH antagonists for IVF, the criteria used for inducing final oocyte maturation during ovarian stimulation vary markedly among investigators, making comparisons of results difficult. Whereas some have used a combination of follicular size and $E_{2}$ levels [17, 18], most studies administered hCG when greater than or equal to three follicles $\geq 17 \mathrm{~mm}$ are present on ultrasound $[2,3]$. Other studies trigger final oocyte maturation in the presence of greater than or equal to three follicles with a maximum diameter of $18 \mathrm{~mm} \mathrm{[19]} \mathrm{or} \mathrm{in} \mathrm{the} \mathrm{presence} \mathrm{of} \mathrm{greater} \mathrm{than} \mathrm{or} \mathrm{equal} \mathrm{to} \mathrm{one}$ follicle of $\geq 18 \mathrm{~mm}$ and three follicles of $\geq 15 \mathrm{~mm}$ [20]. In contrast, we used the presence of two mature follicles with a mean diameter $>18 \mathrm{~mm}$ with two additional follicles $\geq 16 \mathrm{~mm}$ as the criterion for hCG administration. Also, previous studies on delaying $\mathrm{hCG}$ administration delayed ovulation triggering for a specific number of days ( 1 or 2 days) $[7,15,16]$. In our study, hCG trigger was delayed between 1 and 4 days, per attending physician discretion. The specific reason for delaying $\mathrm{hCG}$ administration could not be determined on chart review; however, the proportion of cycles that were delayed were not significantly different among the two physicians that performed most of the oocyte retrievals during the study pe$\operatorname{riod}(\mathrm{EP} \mathrm{52/126} \mathrm{(41.3 \% )} \mathrm{and} \mathrm{FY} \mathrm{26/64} \mathrm{(40.6 \% )).}$

The development of moderate to severe ovarian hyperstimulation syndrome (OHSS) is a concern when further stimulation continues in patients that have met the criteria for hCG as stipulated by individual units' protocol. In our study, 3.8\% of our patients developed moderate to severe OHSS in delayed 
group, compared to none in the non-delayed group, although the difference was not statistically significant. At the time hCG was withheld in group 1 , the $\mathrm{E}_{2}$ was significantly lower compared to group 2 . This situation was reversed by the time hCG was eventually administered to patients in group 1 . It is conjectural to speculate whether the OHSS that developed in this group could have been prevented given that no advantage was gained by delaying administration of $\mathrm{hCG}$ in this group in terms of CPR and LBRs after accounting for all the confounding variables. The fear that continuing ovarian superovulation and further rise in serum $E_{2}$ may put patients at high risk of OHSS should be another reason to avoid further ovarian stimulation once the criteria for administrating hCG trigger are reached.

Our study results are at variance with the finding of an earlier randomized study that suggested that a window of at least 3 days exists during which hCG can be given [7] without compromising the LBR. We found that an increase in the number of days of superovulation decreased the odds of livebirth by $35 \%$ after adjusting for confounding variables. Therefore, the practice of delaying hCG to allow further growth of medium size follicles when criteria for administering hCG has been met should be discouraged. Two studies [4, 6] have suggested that only approximately $31-38 \%$ of oocytes become useable embryos for transfer or cryopreservation, and that LBR is not necessarily increased when more than 15 oocytes are retrieved. The median number of oocytes collected in our study is in keeping with this suggestion. Therefore, the aim should be to stimulate patients with intent to collect not more than this number of oocytes. However, others have gone further and suggested that with good IVF laboratory conditions if a patient reaches embryo transfer the number of eggs retrieved should not affect the likelihood of pregnancy outcome [21].

A limitation of this study is its retrospective design with potential for missing data. In addition, no systematic criteria were applied to determine when to and who to delay before hCG was administered as this was done at the discretion of the attending physician taking care of the patient. However, the proportions of cycles that were delayed were not different among physicians who performed the patients' oocyte collections. Another limitation is the inability to evaluate the criteria used to determine the day of transfer. However, the default day of transfer at the time of this study was day $3(180,90.0 \%)$ with only a few patients having their transfer on day $2(13$, $6.5 \%)$ and day $5(7,3.5 \%)$. This variable did not influence CPR and LBR when included in the logistic regression model.

In conclusion, slightly asynchronous follicular development does not compromise CPR and LBR in IVF/ICSI cycles. Delaying hCG administration to allow further growth of medium-sized follicles should be discouraged as it added further days of superovulation and increases cost of treatment without improvement in CPR and LBR. Only maternal age and the number of days for superovulation influenced CPR and LBR, respectively, in this study.

\section{Compliance with ethical standards}

Conflict of interest The authors declare that they have no conflict of interest.

\section{References}

1. de Mouzon J, Goossens V, Bhattacharya S, Castilla JA, Ferraretti AP, European Ivf-monitoring Consortium ftESoHR, et al. Assisted reproductive technology in Europe, 2006: results generated from European registers by ESHRE. Hum Reprod. 2010;25(8):1851-62.

2. Kolibianakis E, Bourgain C, Albano C, Osmanagaoglu K, Smitz J, Van Steirteghem A, et al. Effect of ovarian stimulation with recombinant follicle-stimulating hormone, gonadotropin releasing hormone antagonists, and human chorionic gonadotropin on endometrial maturation on the day of oocyte pick-up. Fertil Steril. 2002;78(5):1025-9.

3. Kolibianakis EM, Albano C, Kahn J, Camus M, Tournaye H, Van Steirteghem AC, et al. Exposure to high levels of luteinizing hormone and estradiol in the early follicular phase of gonadotropinreleasing hormone antagonist cycles is associated with a reduced chance of pregnancy. Fertil Steril. 2003;79(4):873-80.

4. Meniru GI, Craft IL. Utilization of retrieved oocytes as an index of the efficiency of superovulation strategies for in-vitro fertilization treatment. Hum Reprod. 1997;12(10):2129-32.

5. Jaffe SB, Jaffe LH, Jewelewicz R. Incidence of severe ovarian hyperstimulation syndrome with extremely elevated serum estrogen levels. Gynecol Obstet Investig. 1993;35(4):222-7.

6. Patrizio P, Sakkas D. From oocyte to baby: a clinical evaluation of the biological efficiency of in vitro fertilization. Fertil Steril. 2009;91(4):1061-6.

7. Tan SL, Balen A, el Hussein E, Mills C, Campbell S, Yovich J, et al. A prospective randomized study of the optimum timing of human chorionic gonadotropin administration after pituitary desensitization in in vitro fertilization. Fertil Steril. 1992;57(6):1259-64.

8. Dimitry ES, Bates SA, Oskarsson T, Margara R, Winston RM. Programming in vitro fertilization for a 5- or 3-day week. Fertil Steril. 1991;55(5):934-8.

9. Abdalla HI, Baber RJ, Leonard T, Kirkland A, Mitchell A, Power $\mathrm{M}$, et al. Timed oocyte collection in an assisted conception programme using GnRH analogue. Hum Reprod. 1989;4(8):927-30.

10. Chen Y, Zhang Y, Hu M, Liu X, Qi H. Timing of human chorionic gonadotropin (hCG) hormone administration in IVF/ICSI protocols using GnRH agonist or antagonists: a systematic review and metaanalysis. Gynecol Endocrinol. 2014;30(6):431-7.

11. Morley L, Tang T, Yasmin E, Hamzeh R, Rutherford AJ, Balen AH. Timing of human chorionic gonadotrophin (hCG) hormone administration in IVF protocols using GnRH antagonists: a randomized controlled trial. Hum Fertil (Camb). 2012;15(3):134-9.

12. Tremellen KP, Lane M. Avoidance of weekend oocyte retrievals during $\mathrm{GnRH}$ antagonist treatment by simple advancement or delay of hCG administration does not adversely affect IVF live birth outcomes. Hum Reprod. 2010;25(5):1219-24.

13. Porter RN, Smith W, Craft IL, Abdulwahid NA, Jacobs HS. Induction of ovulation for in-vitro fertilisation using buserelin and gonadotropins. Lancet. 1984;2(8414):1284-5.

14. Diedrich K, Diedrich C, Santos E, Zoll C, al-Hasani S, Reissmann $\mathrm{T}$, et al. Suppression of the endogenous luteinizing hormone surge 
by the gonadotrophin-releasing hormone antagonist Cetrorelix during ovarian stimulation. Hum Reprod. 1994;9(5):788-91.

15. Clark L, Stanger J, Brinsmead M. Prolonged follicle stimulation decreases pregnancy rates after in vitro fertilization. Fertil Steril. 1991;55(6):1192-4.

16. Kolibianakis EM, Albano C, Camus M, Tournaye H, Van Steirteghem AC, Devroey P. Prolongation of the follicular phase in in vitro fertilization results in a lower ongoing pregnancy rate in cycles stimulated with recombinant follicle-stimulating hormone and gonadotropin-releasing hormone antagonists. Fertil Steril. 2004;82(1):102-7.

17. Albano C, Felberbaum RE, Smitz J, Riethmuller-Winzen H, Engel J, Diedrich K, et al. Ovarian stimulation with HMG: results of a prospective randomized phase III European study comparing the luteinizing hormone-releasing hormone (LHRH)-antagonist cetrorelix and the LHRH-agonist buserelin. European Cetrorelix Study Group. Hum Reprod. 2000;15(3):526-31.

18. Felberbaum RE, Albano C, Ludwig M, Riethmuller-Winzen H, Grigat M, Devroey P, et al. Ovarian stimulation for assisted reproduction with HMG and concomitant midcycle administration of the GnRH antagonist cetrorelix according to the multiple dose protocol: a prospective uncontrolled phase III study. Hum Reprod. 2000;15(5):1015-20.

19. Garcia-Velasco JA, Isaza V, Vidal C, Landazabal A, Remohi J, Simon C, et al. Human ovarian steroid secretion in vivo: effects of GnRH agonist versus antagonist (cetrorelix). Hum Reprod. 2001;16(12):2533-9.

20. de Jong D, Macklon NS, Fauser BC. A pilot study involving minimal ovarian stimulation for in vitro fertilization: extending the "follicle-stimulating hormone window" combined with the gonadotropin-releasing hormone antagonist cetrorelix. Fertil Steril. 2000;73(5):1051-4.

21. Yoldemir T, Fraser IS. The effect of retrieved oocyte count on pregnancy outcomes in an assisted reproduction program. Arch Gynecol Obstet. 2010;281(3):551-6. 